\title{
Energie und Mobilität - Bestandsaufnahme und Entwicklungen
}

\section{W. Gawlik}

Online publiziert am 24. Jänner 2017

(C) Springer Verlag Wien 2017

Mit der Einschätzung „Die Elektricität wird im kommenden Jahrhundert die bewegende Kraft sein für elegante Fiaker und für Luxuswagen in Städten" lag die Zeitschrift DER MOTORWAGEN im Jahr 1898 nicht vollständig richtig. Entgegen den Entwicklungen im öffentlichen Nah- und Fernverkehr, der bereits weitgehend elektrifiziert ist, spielt die Elektromobilität beim motorisierten Individualverkehr derzeit noch eine untergeordnete Rolle.

Das liegt insbesondere daran, dass aufgrund der begrenzten Ladeleistungen und der vergleichsweise geringen Reichweiten die Vorteile der Elektromobilität gegenüber Mobilität mit Verbrennungsmotoren noch nicht ausschlaggebend sind. Beim Tankvorgang mit Benzin oder Diesel findet umgerechnet ein "Ladevorgang" des Tanks mit
25 bis 30 MW statt, während die Ladeleistung beim Normalladen einer Batterie in der Größenordnung von $10 \mathrm{~kW}$, beim beschleunigten Laden bei $22 \mathrm{~kW}$ und beim Schnellladen bei $50 \mathrm{~kW}$ und mehr liegt. Insofern stellt sich die Frage, ob der Ladevorgang nicht besser aus dem Fahrzeug heraus verlagert werden sollte bzw. ob andere Energieträger für Mobilitätsanwendungen zu bevorzugen wären.

Insbesondere muss aber nicht nur bei der Energieversorgung für die Mobilität, sondern beim Mobilitätsverhalten selbst angesetzt werden.

Der Vortrag nimmt eine Bestandsaufnahme vor und zeigt einige historische und gegenwärtige (Fehl-)Entwicklungen. 